\title{
Förderung der Justiz in Afrika: Austauschprogramm zwischen Baden-Württemberg und zentralafrikanischen Staaten
}

\author{
Harald Sippel ${ }^{*}$
}

\section{Hintergrund}

Wissenschaftliche Untersuchungen, die sich eingehend mit der Funktionsfähigkeit, der Akzeptanz und der Förderung der Justiz in afrikanischen Staaten befassen, werden im Rahmen der Jahrestagungen der Gesellschaft für afrikanisches Recht e.V. und in dieser Zeitschrift seit etlichen Jahren einem stets an dieser vielseitigen Thematik interessierten Publikum vorgestellt. ${ }^{1}$ An diese Erkenntnisse anknüpfend entstand der Gedanke, sie zumindest teilweise auch in der Praxis umzusetzen und ein Austauschprogramm für bereits berufserfahrene junge Juristinnen und Juristen aus Burundi, der Demokratischen Republik Kongo und Ruanda mit der Justiz in Baden-Württemberg zu etablieren.

\section{Kooperation}

Möglich gemacht wird das Austauschprogramm erst durch die enge Zusammenarbeit verschiedener Institutionen und Personen. Dies sind nicht nur die Hauptbeteiligten, nämlich die afrikanischen Juristinnen und Juristen sowie ihre staatlichen und privaten Arbeitgeber und die Justiz sowie andere juristische Institutionen in Baden-Württemberg, sondern auch die Robert Bosch Stiftung in Stuttgart als fördernde Institution und die Gesellschaft für afrikanisches Recht e.V. als antragstellende Institution.

Von afrikanischer Seite nehmen an dem Austauschprogramm junge, bereits berufserfahrene Juristinnen und Juristen mit sehr guten fachlichen Qualifikationen, aktivem Engagement für die Realisierung rechtsstaatlicher Strukturen in ihren Heimatländern und Interesse am Austausch mit europäischen Partnern teil.

Über das Bekenntnis der baden-württembergischen Landesregierung im Koalitionsvertrag $^{2}$ für die laufende Legislaturperiode 2011 bis 2016, sich für „,weltweit mehr Gerechtigkeit durch Bildung und Partnerschaft" einsetzen zu wollen und insbesondere die bestehende

* Dr. Harald Sippel ist Privatdozent an der Rechts- und Wirtschaftswissenschaftlichen Fakultät der Universität Bayreuth (Deutschland).

1 So war etwa „Justiz in Afrika“ das Leitthema der 33. Jahrestagung der Gesellschaft für afrikanisches Recht in Loccum, Deutschland, vom 30. November bis zum 2. Dezember 2007. Veröffentlicht sind die Ergebnisse in: Recht in Afrika 11 (2008), S. 1-185, und in: Recht in Afrika 12 (2009), S. 21-67.

2 Siehe: http://www.baden-wuerttemberg.de/fileadmin/redaktion/dateien/PDF/Koalitionsvertrag-web. pdf (abgerufen am 10.9.2014). 
Partnerschaft mit Burundi zu festigen und auszubauen, wird bereits an anderer Stelle in dieser Zeitschrift berichtet. ${ }^{3}$ Der Aufbau des juristischen Austauschprogramms ist ein schönes Beispiel für die praktische Umsetzung des vorgenannten politischen Vorhabens.

Die Robert Bosch Stiftung versteht sich nach eigenem Bekunden unter anderem auch als fördernde Organisation, „die es Dritten ermöglicht, interessante Ansätze ... zur Bewältigung gesellschaftlicher Aufgaben im In- und Ausland zu entwickeln und umzusetzen. “4 Obgleich Afrika nicht geografischer Förderschwerpunkt der Stiftung ist, unterstützt sie dort in den südlich der Sahara gelegenen Staaten Projekte, um ,wissenschaftliche Talente zu fördern, die zur Lösung der ökologischen, ökonomischen und sozialen Probleme der ärmsten Länder" beizutragen geeignet sind. ${ }^{5}$ Diese Zielvorgabe der Robert Bosch Stiftung deckt sich mit der Intention das juristischen Austauschprogramms, durch die Fortbildung der afrikanischen Juristinnen und Juristen in Baden-Württemberg die Umsetzung rechtsstaatlicher Strukturen in den Entsendeländern zu unterstützen und damit den sozialen Zusammenhalt in der afrikanischen Gesellschaft zu stärken.

Die Gesellschaft für afrikanisches Recht e.V. schließlich sieht sich bereits aufgrund ihres Satzungszwecks, „die Kenntnis der Rechtsordnungen des gesamten afrikanischen Raums ... zu verbreiten und deren vergleichendes Studium zu fördern"“6 dazu veranlasst, das Austauschprojekt als antragstellende Institution zu unterstützen und zu begleiten. Verantwortlich für die Projektbearbeitung ist mit Hartmut Hamann ein Vorstandsmitglied der Gesellschaft. $^{7}$

\section{Austauschprogramm}

Das Ziel des Projekts ist vor allem der Aufbau eines Netzwerkes aus afrikanischen und deutschen Juristinnen und Juristen, das die Umsetzung rechtsstaatlicher Strukturen in den afrikanischen Partnerländern unterstützt und die Beziehungen zwischen Baden-Württemberg und den Partnerländern fördert. Dies erfolgt in dieser Projektphase insbesondere durch die Vermittlung von Wissen und Erfahrungen seitens deutscher Juristinnen und Juristen an ihre afrikanischen Gäste. Konkret geschieht dies im Rahmen eines zweiwöchigen Aufenthalts von insgesamt sieben Juristinnen und Juristen aus der Demokratischen Republik Kongo, Burundi und Ruanda in Baden-Württemberg im Oktober 2014. Während dieser Zeit wird jedem der afrikanischen Gäste eine Mentorin bzw. ein Mentor aus dem Bereich der baden-württembergischen Zivil- oder Strafgerichtsbarkeit oder der Staatsanwaltschaft zugeordnet. Die afrikanischen Juristinnen und Juristen sollen während eines einwöchigen Gast-

3 Siehe den Bericht von Sebastian Ambros in dieser Ausgabe.

4 Siehe: http://www.bosch-stiftung.de (Stand 17.8.2014).

5 Siehe: http://www.bosch-stiftung.de (Stand 17.8.2014).

6 Siehe $\S 2$ Abs. 1 der Satzung der Gesellschaft für afrikanisches Recht e.V.

7 Prof. Dr. Hartmut Hamann ist unter folgender Adresse via E-Mail erreichbar: hamann@hamann-leg al.de. 
aufenthaltes im Tätigkeitsbereich der Mentorin bzw. des Mentors wichtige Einblicke in die Strukturen und Abläufe der deutschen Justiz erhalten. In der zweiten Woche des Gastaufenthaltes sind juristische Schulungen $\mathrm{zu}$ verschiedenen, durchaus auch praxisrelevanten Themen (z.B. gewerblicher Rechtsschutz, Urheber und Presserecht, Aufgaben der Generalstaatsanwaltschaft, Aufsicht über die Staatsanwaltschaften, internationale Rechtshilfe im Strafrecht, juristische Ausbildung in Deutschland, Steuersystem in Deutschland, Exekutive im föderalen System, Verfassungsrecht, Europarecht, Internationales Straf- und Völkerstrafrecht) und ein Rahmenprogramm unter anderem mit Besuch des Bundesverfassungsgerichts sowie des Bundesgerichtshofs in Karlsruhe, des Staatsministeriums und des Justizministeriums Baden-Württembergs, des baden-württembergischen Landeskriminalamts, der Justizvollzugsanstalt Heimsheim, der Zentralen Stelle der Landesjustizverwaltungen zur Aufklärung nationalsozialistischer Verbrechen in Ludwigsburg sowie einer Hauptverhandlung des Schöffengerichts beim Amtsgericht Stuttgart-Bad Cannstatt geplant.

\section{Erwartete Ergebnisse und Perspektiven}

Die Teilnehmerinnen und Teilnehmer aus den afrikanischen Partnerländern erhalten durch das Austauschprogramm die Möglichkeit, die Strukturen der baden-württembergischen Justiz kennenzulernen und sich in praxisrelevanten Rechtsgebieten fortzubilden. Insbesondere durch die Einbindung von Mentorinnen und Mentoren werden sie aufgrund der gewonnenen Erfahrungen und mittels des dabei entstandenen Netzwerks mit anderen Juristinnen und Juristen bei der Umsetzung rechtsstaatlicher Strukturen in ihren jeweiligen Heimatländern unterstützt. Dieses Netzwerk ist geeignet, die Beziehungen zwischen der Bundesrepublik Deutschland und den afrikanischen Partnerländern zu fördern. Auf deutscher Seite wird den partizipierenden Justiz- und Lehrpersonen die Möglichkeit eröffnet, ihren Erfahrungshorizont in rechtsvergleichender Hinsicht zu erweitern und am vorgenannten Netzwerk teilzuhaben, was zugleich der Weiterentwicklung der eigenen Arbeitspraxis dient.

Sollte sich das Austauschprogramm in diesem Jahr als erfolgreich erweisen, besteht die Absicht, es auch im Jahr 2015 zu organisieren. Dann könnte eine Ausweitung auf andere afrikanische Partnerländer und die Einbeziehung weiterer deutscher Bundesländer erfolgen. Ideal wäre auch ein Gastaufenthalt deutscher Juristinnen und Juristen bei Institutionen der Justiz afrikanischer Partnerländer.

Letztlich haben darüber aber die Beteiligten des Projekts nach der Evaluierung des Austauschprogramms zu entscheiden. In dieser Hinsicht sehen wir bereits jetzt einem Bericht seitens der afrikanischen Teilnehmerinnen und Teilnehmer über ihre Erfahrungen mit dem Austauschprogramm in Baden-Württemberg im Oktober 2014 erwartungsvoll entgegen, der möglichst zeitnah in dieser Zeitschrift erscheinen möge. 


\section{Impressum}

Recht in Afrika - Law in Africa - Droit en Afrique - Zeitschrift der Gesellschaft für afrikanisches Recht

Herausgeber: Gesellschaft für afrikanisches Recht e.V., vertreten durch den Vorsitzenden des Vorstandes, Herrn Dr. Harald Sippel, Barbarossastraße 31, 09112 Chemnitz

Schriftleitung: RA Prof. Dr. Hartmut Hamann, Falkertstraße 82, D-70193 Stuttgart Telefon: +49 (o) 711 120950-30 | Fax : +49 (0) 711 120950-50

Mail: hamann@hamann-legal.de

Erscheinungsweise: 4 Ausgaben pro Jahr

Druck und Verlag: Nomos Verlagsgesellschaft mbH \& Co. KG - Waldseestr. 3-5 D-76530 Baden-Baden. Telefon +49-7221-2104-0 / Fax 49-7221-2104-27

E-Mail:nomos@nomos.de

Anzeigen: sales friendly Verlagsdienstleistungen - Inh. Frau Bettina Roos - Pfaffenweg 15 - D-53227 Bonn Telefon +49-228-97898-o / Fax +49-228-97898-20 E-Mail:roos@sales-friendly.de

Urheber- und Verlagsrechte: Die Zeitschrift und alle in ihr enthaltenen einzelnen Beiträge sind urheberrechtlich geschützt. Jede Verwertung außerhalb der engen Grenzen des Urheberrechtsgesetzes ist ohne Zustimmung des Verlages unzulässig. Das gilt insbesondere für Vervielfältigungen, Übersetzungen, Mikroverfilmungen und die Einspeicherung und Verarbeitung in elektronischen Systemen. Der Nomos Verlag beachtet die Regeln des Börsenvereins des Deutschen Buchhandels e.V. zur Verwendung von Buchrezensionen.

ISSN 2363-6270

\section{Nomos}

